\title{
MISSING SOLAR ULTRAVIOLET OPACITY AND DIATOMIC MOLECULES
}

\author{
S. P. TARAFDAR and M. S. VARDYA
}

Tata Institute of Fundamental Research, Bombay, India

\begin{abstract}
The solar atmospheric model calculations incorporating absorption by hydrogen and metals and Rayleigh scattering by hydrogen give larger specific intensity in the solar ultraviolet spectrum than that observed. This discrepancy has been interpreted as due to some unknown source(s) of opacity in this spectral region. We have, therefore, compared the absorption coefficient due to bands of nine diatomic molecules with that of metals and hydrogen in the ultraviolet spectral region at temperatures and pressures appropriate to the solar photosphere. The molecular band absorptions due to $\mathrm{A}-\mathrm{X}$ bands of $\mathrm{CO}, \mathrm{SiO}$ and $\mathrm{OH}$ and to $\mathrm{B}-\mathrm{X}$ band of $\mathrm{CH}$ have been found to be larger than or equal to the total absorption by metals and hydrogen below $4000 \AA$ and may be able to account for the missing opacity except possibly between $2500 \AA$ and $3000 \AA$. Whether absorption due to some other molecular band or bands is responsible for the opacity in this wavelength interval is not certain.
\end{abstract}

* For the detailed paper, see Astrophys. J. 171 (1972), 185. 